\title{
Three-dimensional high-resolution quantitative microscopy of extended crystals
}

\author{
P. Godard ${ }^{1, \uparrow}$, G. Carbone ${ }^{2}$, M. Allain', F. Mastropietro ${ }^{2,3}$, G. Chen ${ }^{4}$, L. Capello ${ }^{5}$, A. Diaz ${ }^{2, \uparrow}$, T.H. Metzger ${ }^{2, \uparrow}$ \\ J. Stang $\left.\right|^{4} \&$ V. Chamard ${ }^{1}$
}

Hard X-ray lens-less microscopy raises hopes for a non-invasive quantitative imaging, capable of achieving the extreme resolving power demands of nanoscience. However, a limit imposed by the partial coherence of third generation synchrotron sources restricts the sample size to the micrometer range. Recently, X-ray ptychography has been demonstrated as a solution for arbitrarily extending the field of view without degrading the resolution. Here we show that ptychography, applied in the Bragg geometry, opens new perspectives for crystalline imaging. The spatial dependence of the three-dimensional Bragg peak intensity is mapped and the entire data subsequently inverted with a Bragg-adapted phase retrieval ptychographical algorithm. We report on the image obtained from an extended crystalline sample, nanostructured from a silicon-on-insulator substrate. The possibility to retrieve, without transverse size restriction, the highly resolved three-dimensional density and displacement field will allow for the unprecedented investigation of a wide variety of crystalline materials, ranging from life science to microelectronics.

\footnotetext{
${ }^{1}$ Institut Fresnel, CNRS, Aix-Marseille University, FST Saint-Jérome, Marseille Cedex 20, 13397, France. ${ }^{2}$ European Synchrotron Radiation Facility, BP220, Grenoble, 38043, France. ${ }^{3}$ CEA/Grenoble, Institut Nanosciences et Cryogénie, Laboratoire SP2M/Nano-structures et Rayonnement Synchrotron, Grenoble Cedex 9, 38054, France. ${ }^{4}$ Institute of Semiconductor and Solid State Physics, Johannes Kepler Universität, Linz 4040, Austria. ${ }^{5}$ Soitec S.A., Parc Technologique des Fontaines, Bernin, 38190, France. †Present addresses: Department of Electrical \& Electronic Engineering, University of Sheffield, S1 3JD, UK (P.G.); Paul Scherrer Institut, 5232 Villigen, Switzerland (A.D.); Max Planck Institute of Colloids and Interfaces, Department of Biomaterials, 14424 Potsdam, Germany (T.H.M.). Correspondence and requests for materials should be addressed to V.C. (email: virginie.chamard@fresnel.fr).
} 
uantitative imaging of strains, dislocations, inclusions and so on becomes in reach with the advances of X-ray coherent diffraction imaging ${ }^{1}$. The last makes use of the far-field intensity pattern produced by a crystal, illuminated by a plane-wave in Bragg conditions. The phase retrieval is ensured by the oversampling condition, which imposes a finite sample size ${ }^{2}$. The inversion is performed with iterative algorithms, based on back-and-forth propagations of the wavefield from the sample to the detector plane (see ref. 3 and references therein). However, its development encounters two main limits. For weak displacement fields where small phase shifts in the exit wave-field are expected (for example, owing to surface strain), the obtained reconstruction does not allow the discrimination between illumination wavefront inhomogeneities and crystal displacement fields ${ }^{4}$. The opposite case of highly heterogeneous strain fields, present in a wide variety of materials (epitaxial and/or embedded nanocrystals, poly-crystalline materials, and so on), is also problematic: investigations are prohibited by the extremely slow convergence of the inversion procedure ${ }^{5-7}$. Bragg ptychography can overcome these limits: it is expected to enable the simultaneous recovering of the sample and illumination functions and its robustness, with regard to strain inhomogeneity, is evidenced from numerical analysis ${ }^{8}$.

Ptychography, originally proposed for electron microscopy applications ${ }^{9}$, makes use of multiple exposures that combine redundant information from partially overlapping illumination areas onto an extended sample ${ }^{10}$. A set of far-field coherent intensity patterns is recorded while the sample is moved through the finite-sized beam spot. The redundancy, resulting from the overlapping condition is the key of the iterative process convergence ${ }^{11}$. It releases the need for an intensity oversampling condition, opening the field of X-ray lens-less microscopy to samples larger than the transverse coherence lengths of the beam ${ }^{12-16}$. Recently, the ptychographical iterative engine (PIE) has been proposed ${ }^{17}$ and further extended ${ }^{18,19}$ to retrieve simultaneously the sample scattering contrast and the illumination function.

Combining ptychography with Bragg diffraction is highly desirable for crystalline imaging. Moreover, Bragg geometry offers a major advantage for ptychography. A small angular exploration along the rocking curve in the vicinity of the chosen Bragg reflection corresponds to a translation of the wave-vector transfer across the Bragg peak. In practice, full three-dimensional (3D) intensity patterns can be recorded within small angular movements of less than a degree ${ }^{20}$ : the illumination volume can be considered as constant along the rocking curve. Hence, instead of handling the two-dimensional (2D) wave-fields for each illumination position and each tomographic angle ${ }^{14}$, Bragg ptychography allows dealing directly with the full 3D intensity patterns, for each illumination position only. This approach drastically reduces computational efforts.

Here we report on the experimental demonstration of Bragg ptychography imaging. To this aim, we have designed an extended crystalline nanostructure from a silicon-on-insulator substrate. The finite-sized coherent beam spot required for the measurement was produced by the coherent illumination of a Fresnel zone plate $(\mathrm{FZP})^{21}$. The 3D Bragg diffraction intensity was measured in the vicinity of the chosen Bragg reflection, as a function of the beam position on the sample. The step size was small enough to ensure the needed high redundancy in the data set. The sample-scattering contrast and the illumination function were simultaneously retrieved from the whole set of intensity data, using a Bragg adapted PIE ${ }^{8}$. The retrieved features exhibited by the $3 \mathrm{D}$ crystalline density and displacement field are discussed below. We expect that this technique, that enables new insights of crystalline nanostructures, will have a key role in materials science.

\section{Results}

$\mathrm{X}$-ray bragg ptychography experimental set-up. Figure 1a shows the schematic layout of the experimental X-ray Bragg ptychography
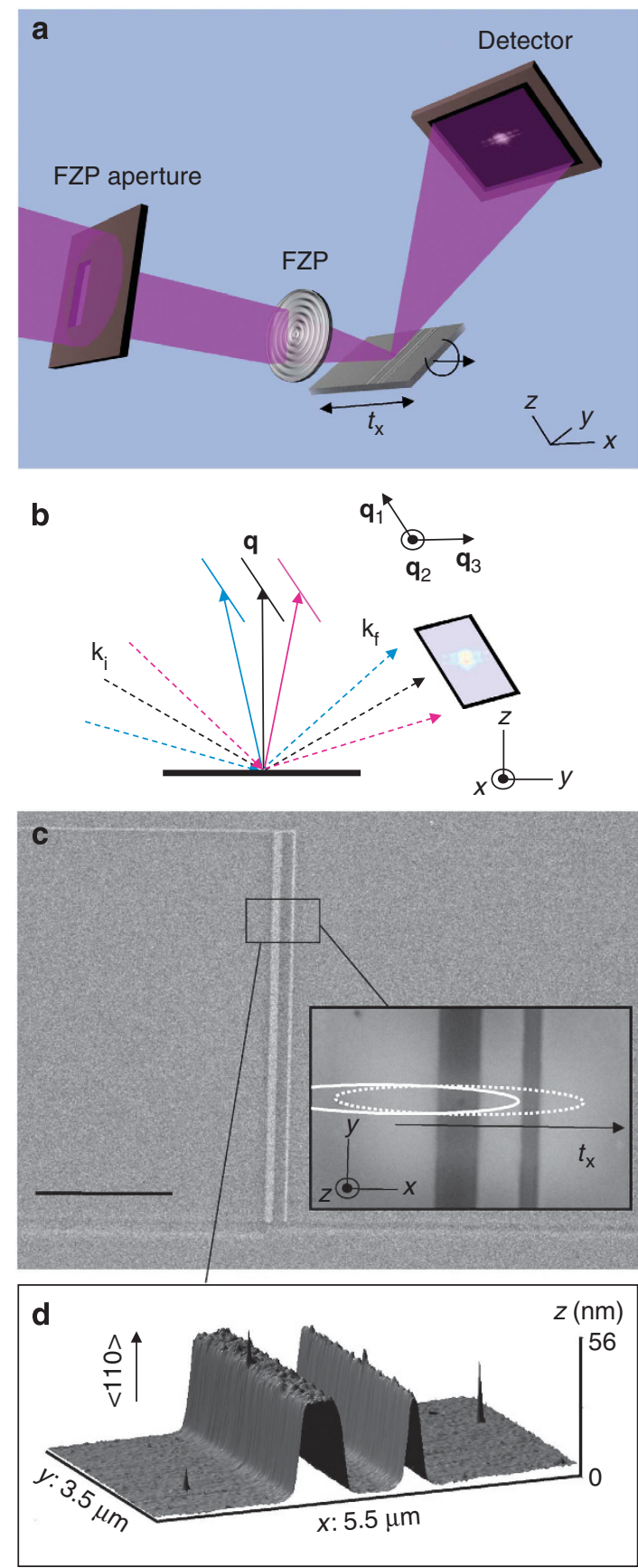

Figure 1 | Bragg ptychography experimental set-up and sample. (a) X-ray Bragg ptychography set-up including the FZP and its slit-defining aperture, the sample stage and the far-field 2D detector (The FZP central stop and the order sorting aperture have been omitted for clarity). The direct space frame $(\mathbf{x}, \mathbf{y}, \mathbf{z})$ is chosen in the sample frame. (b) Sketch of the 3D intensity acquisition in Bragg geometry and the associated reciprocal space vectors $\mathbf{q}_{1}, \mathbf{q}_{2}$ and $\mathbf{q}_{3}$. (c) Scanning electron micrograph of the lines patterned on the Silicon-on-insulator substrate (Scale bar: $10 \mu \mathrm{m}$ ). The zoomed region (inset) emphasizes the area investigated during the ptychography scan. The translation direction $t_{x}$ is shown together with the two first illumination areas (white ellipses). (d) The same region as seen by atomic force microscopy.

set-up. The finite-sized beam spot needed for the ptychography scan results from the focalization of a fully coherent monochromatic beam using a FZP22. For this purpose, the FZP aperture is reduced so that the illumination area matches the beam transverse coherence 


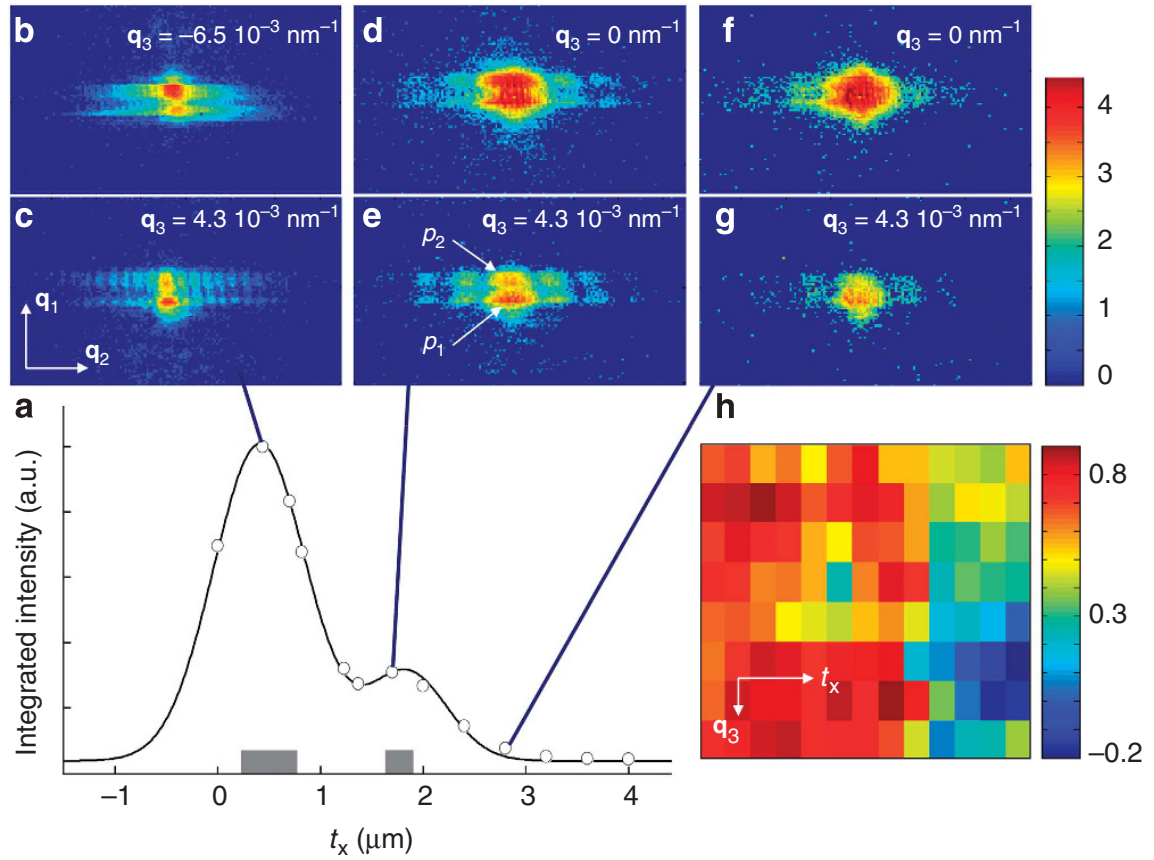

Figure 2 | Bragg ptychography experimental data. (a) Integrated intensity values as a function of the beam translation $t_{\mathrm{x}}$ : the two maxima correspond to the two Si lines, which are schematically indicated (grey rectangles). The line is a guide to the eye. (b-g) 2D coherent intensity patterns measured at the Si 220 reflection for different $q_{3}$ values (indicated on the graphs) and different positions of the focused beam onto the double line crystalline sample. The corresponding $t_{\mathrm{x}}$ values are indicated in (a). A splitting of the Bragg peak is observed along $\mathbf{q}_{1}$, emphasized in (e) by the white arrows and the respective peak labels, $p_{1}$ and $p_{2}$. The intensity colour scale is logarithmic. The intensity has been multiplied by 6 in $(\mathbf{d}, \mathbf{e})$ and by 25 in $(\mathbf{f}, \mathbf{g})$, for clarity. The reciprocal space scale is indicated in the figures where $\left|q_{1}\right|=\left|q_{2}\right|=5 \times 10^{7} \mathrm{~m}^{-1}$. (h) Splitting contrast $C_{\mathrm{s}}=1-\left(I_{\min } / I_{p}\right)$ as a function of $\mathbf{q}_{3}$ and $t_{\mathrm{x}}: I_{\min }$ is the mean intensity value at the peak splitting minimum and $I_{p}$ is the mean intensity at the $p_{1}$ and $p_{2}$ positions. Therefore, $C_{s}=1$ corresponds to a full splitting. In (h), the abrupt change along $t_{x}$ evidences that the Bragg splitting is linked to the sample structure and occurs preferentially at the large line position. The corresponding colour scale is given on the right. The scale is $\left|\mathbf{q}_{3}\right|=2.2 \times 10^{7} \mathrm{~m}^{-1}$.

lengths ${ }^{23}$. As the FZP central part is occluded by a beam stop to avoid direct beam contribution, the aperture is shifted laterally. The sample is oriented in Bragg condition with its surface normal lying in the vertical plane and inclined with respect to the incident beam. The $3 \mathrm{D}$ coherent intensity pattern is measured along the rocking curve (Fig. 1b) at each beam translation $t_{\mathrm{x}}$. The $3 \mathrm{D}$ acquisition is repeated while the sample is moved along the horizontal direction, across the beam. The chosen translation step is about $300 \mathrm{~nm}$. According to our observations with limited FZP aperture, the spotsize full-width-at-half-maximum of the field at the focus should be about $200 \times 600 \mathrm{~nm}^{2}$, along the vertical and horizontal directions, respectively. The Bragg geometry induces a further increase of the beam footprint on the sample, to about $500 \times 600 \mathrm{~nm}^{2}$ along the beam and in horizontal direction, respectively. Hence, an overlapping of at least $50 \%$ is ensured along the scanning direction. With this set-up, a total of about $10^{8}$ photons per second is expected at the focus.

Sample description. The sample, patterned from a Silicon-insulator wafer is composed of two $\mathrm{Si}\langle 110\rangle$ lines of about $40 \mathrm{~nm}$ height and of 520 and $260 \mathrm{~nm}$ width, respectively. The separation between the lines results in an edge-to-edge distance of $1.7 \mu \mathrm{m}$. The line length is about $30 \mu \mathrm{m}$, much larger than the beam footprint. Figure $1 \mathrm{c}, \mathrm{d}$ shows direct characterizations of the sample. The areas delimited by the white ellipses correspond to the first positions of the beam during the scan.

Data analysis. The different crystalline orientation of the sample substrate ensures that the chosen symmetric 220 Bragg reflection arises from the diffraction by the lines only. In Figure $2 \mathrm{a}$, the integrated intensity is plotted as a function of $t_{\mathrm{x}}$. The two maxima correspond to the scattering signals of the two lines. Figure $2 \mathrm{~b}-\mathrm{g}$ shows typical detector frames taken at different $t_{\mathrm{x}}$ values and different reciprocal space positions. Interference fringes are observed along the direction perpendicular to the lines. Their frequency, related to the inverse line width, varies with $t_{\mathrm{x}}$. The visibility contrast is estimated to about $70 \%$. The continuity and reproducibility of the observed features give a strong evidence of the absence of beam drift or radiation damage. From these data, one notices two interesting features. (1) The simultaneous presence of interferences with high and low frequencies, which result from the scattering by the large and small Si lines, is an evidence of the simultaneous illumination of the two lines. It is a consequence of a beam spot broader than expected. This is further confirmed by the integrated intensity profile of Figure $2 \mathrm{a}$, where the line-related maxima are much larger than the line widths, as a result of the convolution with the beam. (2) More surprising, a split in the Bragg peak along the Bragg vector direction is observed, which leads to a double peak (labelled $p_{1}$ and $p_{2}$ in Figure $2 \mathrm{e}$ ). Figure $2 \mathrm{~h}$ allows a further characterization of the peak splitting from the calculation of the splitting intensity contrast, whose behaviour as a function of $t_{\mathrm{x}}$ clearly shows that the splitting is associated to the large line only. Peak splitting is an usual signature for phase shift in crystal. Final explanations about these features (beam width and Bragg peak splitting) will arise from the Bragg ptychography reconstructions.

$3 \mathrm{D}$ reconstruction of the crystalline scattering contrast. The whole 3D intensity set is analysed with our Bragg ptychography iterative engine, adapted for Bragg diffraction and allowing for the retrieval of the crystal scattering contrast and the illumination function. As usual for the Bragg diffraction case, the scattering contrast is described by a complex-valued function, whose amplitude is associated with the crystal density and whose phase is the 

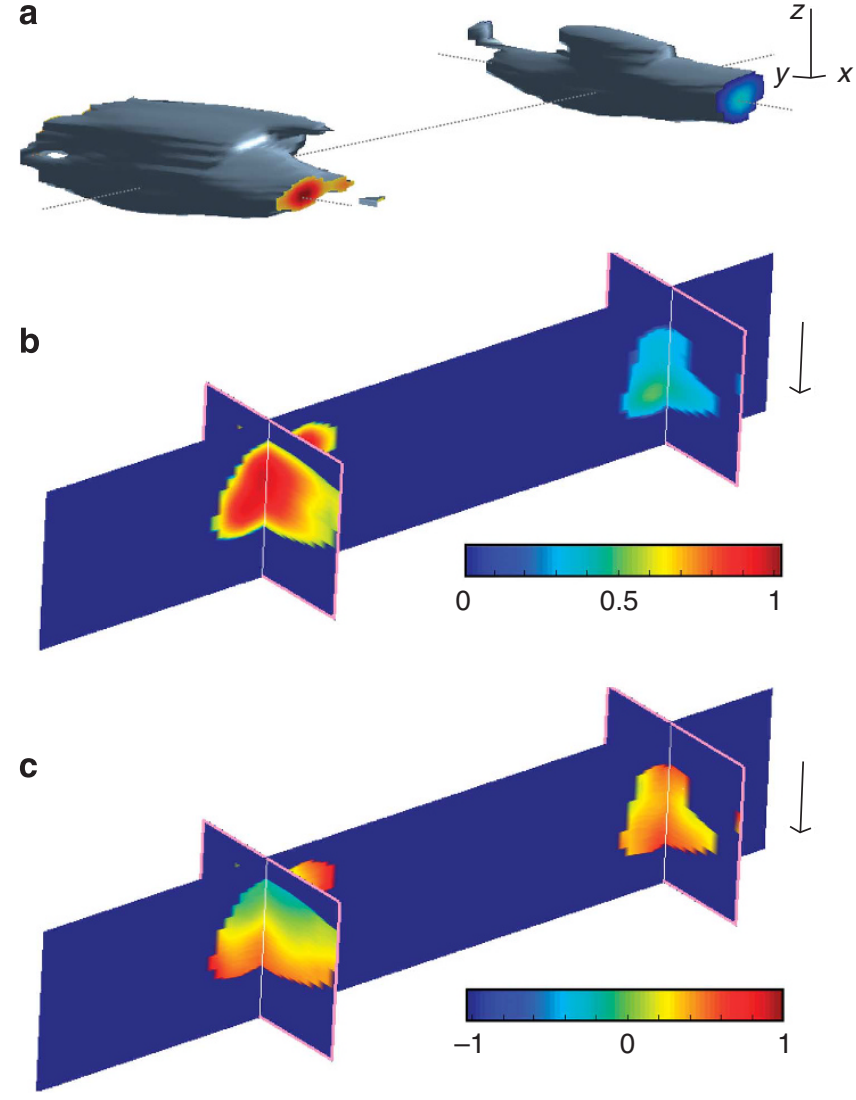

Figure 3 | 3D reconstruction of the double-line crystal. The ptychographical iterative algorithm is used to retrieve the crystal scattering contrast, shown here in an orthogonal space. (a) Reconstructed density represented with a 50\% iso-surface for each line. The dotted grey lines are a guide to the eye. (b) 2D cross-sectional views of the sample density and (c) phase (in rad.). In (a-c), the black lines represent a 40-nm length; The vertical axis has been stretched out for clarity. The colour scales are given in the figures.

projection of the displacement field on the Bragg vector ${ }^{24}$. As a consequence, the 220 Bragg reflection ptychography reconstruction is expected to image only the features associated to the $\mathrm{Si}\langle 110\rangle$ crystal, that is, the two lines without the substrate. The retrieved sample is shown in Figure 3a-c. In Figure 3a, both lines are retrieved with shape, height and width in agreement with the direct characterizations of Figure 1c,d. The finite extent along the line axis corresponds to the finite illumination of the X-ray beam spot. The internal cross-sections of Figure $3 \mathrm{~b}$ exhibit a satisfying homogeneous density. However, the density value is different for the two lines. The smaller value observed for the small line is probably resulting from an interplay between the density and the resolution, which is locally degraded: the resolution actually scales with the invert range of the reciprocal space signal, that is less intense for the thinner line. The phase behaviour is shown on cross-sectional views through the lines. For the small line, the phase is homogeneous, as expected for a strain-free crystal. On the contrary, we observe two constant phase domains in the other line, corresponding to a phase shift of about $0.7 \mathrm{rad}$. The presence of these two phases, observed only in the large crystalline line, finally explains the Bragg peak splitting, evidenced in Figure $2 \mathrm{~b}-\mathrm{d}, \mathrm{h}$. This phase behaviour is the consequence of the coexistence of two crystalline domains, each of them unstrained. These defects have been reported in the literature for crystals produced by Silicon-on-insulator technologies (see ref. 25 and references therein).
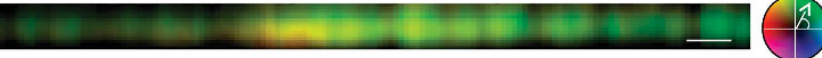

Figure 4 | Reconstruction of the illumination wavefield. The retrieved illumination function is shown in the plane perpendicular to the X-ray beam propagation. On the right, the colour code used for the colour rendition of the complex-valued function is indicated: the length of the arrow and the angle correspond to the amplitude (linear scale) and phase values, respectively (Scale bar: $200 \mathrm{~nm}$ ). The amplitude fluctuations are classical artefacts arising from intensity normalization and translational periodicity of $t_{\mathrm{x}}{ }^{14}$.

Reconstruction of the illumination wavefield. Finally, the retrieved illumination function is shown in Figure 4. It is taken at the sample position in the plane perpendicular to the beam propagation. The vertical beam size is in good agreement with our estimation of the focus spot size. In the horizontal direction, the retrieved spot is asymmetric and its width matches our expectations from the experimental data analysis, which anticipated an horizontally extended beam spot. These two characteristics, horizontal asymmetry and broadening, are typical effects observed when the central stop partly occludes the rectangular aperture at the $\mathrm{FZP}^{21}$. It results from a non-intentional horizontal offset of the FZP aperture position.

\section{Discussion}

Bragg ptychography like other Bragg coherent diffraction imaging methods is expected to provide unrevealed views of $3 \mathrm{D}$ crystalline materials, including density and displacement field. The most comparable microscopy method is certainly transmission electron microscopy, which makes use of electron diffraction to provide highly spatially resolved images of nanocrystals. However, it requires a thinning down of the sample, which restricts its use to structures with a thickness smaller than about 100 nanometers. In addition, the sample preparation is likely to modify the crystalline internal displacement field. Scanning transmission electron microscopy and atomic force microscopy deliver images with resolution in the nanometer range but are only sensitive to topological aspects. In this context, confronting the 3D images obtained from Bragg coherent diffraction approaches to other well-established techniques is a highly difficult task. The sample we used in our Bragg ptychography approach was originally designed for Bragg Fourier transform holography ${ }^{26}$. This approach makes use of a reference crystal located nearby the object crystal to image, which thereby directly allows encoding the phase in the intensity pattern. Hence, a single inverse Fourier transform of the 3D intensity hologram allows the retrieval of the object image. The reconstruction image is the convolution of the object crystal exit field with the reference crystal exit field. The retrieved quantity, obtained from the $3 \mathrm{D}$ diffraction pattern measured at $t_{\mathrm{x}}=1.25 \mu \mathrm{m}$ is shown in Figure 5. The density (Fig. 5a) exhibits height and width in perfect agreement with the dimensions of the crystalline object, while the fine details of the shape are smoothed out by the convolution process. The linear decrease of the density, from a maximum value located in the centre of the object arises from the convolution process as well. The phase (Fig. 5b) shows slow variations with a phase shift in the $1 \mathrm{rad}$ range from the top to the bottom. The good quality of this reconstruction testifies the quality of the measurements. In particular, the presence of a holographic signal confirms the extent of the broadened illumination function over the two Si lines for this probe position. Although this image can not directly be compared with the Bragg ptychography image, the Fourier transform holography results are consistent with the ptychography image. The validity of our 3D Bragg ptychography approach is further testified by the numerical simulation of the whole problem, using detection parameters, sample and illumination functions corresponding to the ones imposed by the experimental conditions. It makes clear that the whole $3 \mathrm{D}$ data set 

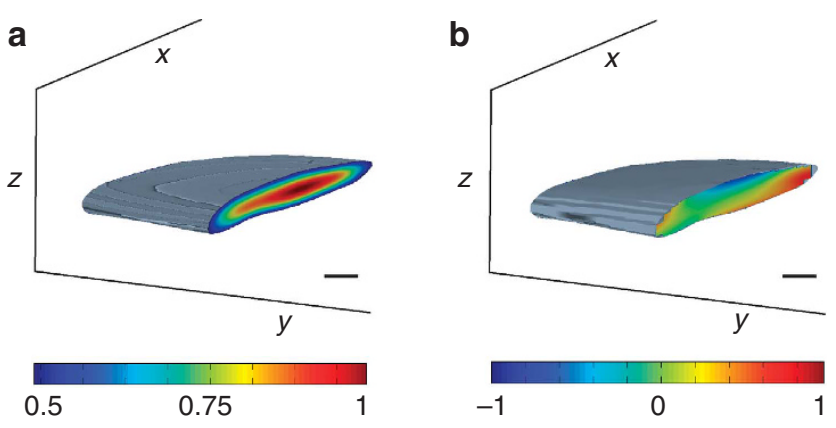

Figure 5 | 3D Bragg Fourier transform holography reconstruction of the crystal. The retrieved crystal scattering contrast, as obtained from Bragg Fourier transform holography analysis, is shown in an orthogonal space. The retrieved image is the convolution of the large Si line by the small reference line. (a) Reconstructed density represented with a 50\% isosurface and (b) reconstructed phase (in rad.): these images are consistent with the retrieved crystal scattering contrasts of (Fig. 3). The colour scales are given in the figures (Scale bars: $50 \mathrm{~nm}$ ).

obtained from the one-dimensional ptychography scan contains all information needed to retrieve the illumination function and the $3 \mathrm{D}$ crystal density and phase.

To conclude, we have experimentally demonstrated that Bragg ptychography is able to produce $3 \mathrm{D}$ quantitative imaging of an extended crystal. In addition to the shape, we obtained an internal view of the density and displacement field. Owing to the possibility offered by Bragg ptychography to image crystals without transverse size restriction, we believe that this technique will allow for new insight and understanding in a large variety of materials, ranging from the most natural ones like bio-crystals up to the most manufactured systems like microelectronics compounds, both being composed of crystals structured on the nanometer scale.

\section{Methods}

Data acquisition. The Bragg ptychography experiment was carried out at the ID01 beamline (European Synchrotron Radiation Facility (ESRF)). The monochromatic beam (wavelength $\lambda=0.154 \mathrm{~nm}$ ) was delivered by a Si-111 monolithic channel-cut monochromator (energy bandwidth of about $1.4 \times 10^{-4}$ ), resulting in a longitudinal coherence length of about $1 \mu \mathrm{m}$. To increase the flux at the sample position, a 200$\mu \mathrm{m}$ diameter Au FZP was placed $129 \mathrm{~mm}$ upstream the sample. A $60-\mu \mathrm{m}$ diameter central stop was placed close to the FZP to avoid direct beam transmission through the FZP central part. The focusing set-up was completed with a $50-\mu \mathrm{m}$ circularorder-sorting aperture located $10 \mathrm{~mm}$ upstream the sample to block the FZP's higher diffraction orders. To ensure a fully coherent beam at the sample position, the focusing optics aperture was set to $80 \times 20 \mu \mathrm{m}^{2}$, corresponding to the vertical and horizontal coherence lengths of the beamline, respectively. Necessarily, the aperture was horizontally shifted to one side of the central stop to ensure a large illumination area onto the FZP. The $\langle 110\rangle$ Si double-line sample was placed in Bragg diffraction geometry (220 reflection, Bragg angle $\theta_{\mathrm{B}}=22.35^{\circ}$ ), with the line axis lying in the scattering plane. The intensity acquisition was performed with a pixel detector (Maxipix, $256 \times 256$ pixels of $55 \times 55 \mu \mathrm{m}^{2}$ size $^{27}$ ) mounted $1.17 \mathrm{~m}$ downstream the sample. The acquisition time was set to $50 \mathrm{~s}$ per frame.

The $3 \mathrm{D}$ intensity is measured as a function of the reduced momentum transfer vector $\mathbf{q}=\mathbf{Q}-\mathbf{G}_{220}$, where $\mathbf{G}_{220}$ is the Bragg vector $\left(\left|\mathbf{G}_{220}\right|=3.3 \times 10^{1} \mathrm{~nm}^{-1}\right)$ and $\mathbf{Q}=\mathbf{k}_{f}-\mathbf{k}_{i}\left(\mathbf{k}_{i}\right.$ (resp. $\left.\mathbf{k}_{f}\right)$ is the incident (resp. diffracted) wavevector with $\left|\mathbf{k}_{i, f}\right|=2 \pi / \lambda$ ). The $\mathbf{q}_{1,2}$ components lie in the detector plane, vertically and horizontally, respectively (Fig. 1b). The $\mathbf{q}_{3}$ direction, which is probed by scanning the incident angle in steps of $0.04^{\circ}$ over an angular range of $0.4^{\circ}$, is perpendicular to $\mathbf{G}_{220}$. These experimental conditions result in a voxel size of $1.9 \times 1.8 \times 22 \times\left(10^{-3}\right)^{3} \mathrm{~nm}^{-3}$. The full set of ptychography data is obtained by scanning the sample along the direction perpendicular to the scattering plane, using a piezo-electric translation stage. The 13 positions cover a total range of about $4 \mu \mathrm{m}$. The whole set-up stability was controlled over hours by a series of X-ray measurements performed before the ptychography scan, showing the absence of beam radiation damage or beam drift in the data set.

Sample preparation. The sample used for this work was produced by e-beam lithography from a continuous $\mathrm{Si}\langle 110\rangle$ layer (180-nm thick) covering a $\mathrm{SiO}_{2}$ film (25-nm thick) onto a $\mathrm{Si}\langle 100\rangle$ substrate (Silicon-on-insulator Smartcut wafer from
Soitec). The two-line structure was defined in a positive photo resist (PMMA E-beam resist AR-P631.04) with a thickness of $130 \mathrm{~nm}$, using a $\mathrm{SF}_{6}$ reactive ion etching to transfer the pattern into the Si layer. The etching depth was chosen to remove completely the $\mathrm{Si}\langle 110\rangle$ layer around the two lines, over an area of $100 \times 100 \mu \mathrm{m}^{2}$.

Phase retrieval. The whole $3 \mathrm{D}$ phase retrieval process is performed in the $\left(\mathbf{q}_{1}, \mathbf{q}_{2}, \mathbf{q}_{3}\right)$ reciprocal space frame and the conjugated $\left(\mathbf{r}_{1}, \mathbf{r}_{2}, \mathbf{r}_{3}\right)$ direct space frame. At the end, the retrieved quantities are transferred into an orthogonal $(\mathbf{x}, \mathbf{y}, \mathbf{z})$ space for plotting.

As usual for iterative algorithms, the initialization requires to built a first guess for the sample scattering contrast, $\rho_{\mathrm{e}}(\mathbf{r})$, and for the illumination volume, $P_{\mathrm{e}}(\mathbf{r})$. Typically, the guessed sample scattering contrast results from the integrated intensity measurement and/or direct characterizations ${ }^{8}$ whereas a $2 \mathrm{D}$ gaussian intensity distribution is used for describing the probe cross-section. The latter is further assumed to be constant along the incident beam propagation. In our work, the constrained PIE (cPIE) is introduced to look for solutions with particular chosen characteristics. The constraint is monitored with a regularization parameter, $\gamma$ $(0 \leq \gamma)$ : in particular, when $\gamma=0$, the cPIE reduces to the usual PIE. The details of the cPIE, which involves a series of $K$ iterations and $N$ sub-iterations, are summarized in the following:

$P_{j}(\mathrm{r})=P_{e}\left(r-R_{j}\right)$ with $1 \leq j \leq N$

$\rho_{1,1}(r)=\rho_{e}(r)$

For $k=1$ to $K$

$$
\begin{aligned}
& \begin{array}{l}
\text { For } j=1 \text { to } N \\
\psi_{k, j}(\mathbf{r})=P_{j}(\mathbf{r}) \rho_{k, j}(\mathbf{r}) \\
E_{k, j}(\mathbf{q})=\left(F \psi_{k, j}\right)(\mathbf{q}) \\
\hat{E}_{k, j}(\mathbf{q})=\sqrt{I_{j}(\mathbf{q})} \frac{E_{k, j}(\mathbf{q})}{\left|E_{k, j}(\mathbf{q})\right|} \\
\hat{\psi}_{k, j}(\mathbf{q})=\left(F^{-1} \hat{E}_{k, j}\right)(\mathbf{q}) \\
\rho_{k, j+1}(\mathbf{r})=\rho_{k, j}(\mathbf{r})-\beta\left[\frac{\left|P_{j}(\mathbf{r})\right|}{\max _{\mathrm{r}}\left|P_{j}(\mathbf{r})\right|\left|P_{j}(\mathbf{r})\right|^{2}+\varepsilon}\right] \\
\quad \times\left(\psi_{k, j}(\mathbf{r})-\hat{\psi}_{k, j}(\mathbf{r})+\gamma \chi(\mathbf{r}) \psi_{k, j}(\mathbf{r})\right)
\end{array}
\end{aligned}
$$

$$
\rho_{k+1,1}(\mathbf{r})=\rho_{k, N+1}(\mathbf{r})
$$

The two first lines describe the initialization in the case where the inversion seeks at refining the sample scattering contrast. $P_{j}$ is the probe at the $R_{j}$ position corresponding to the $j$-th measurement and $N$ is the total number of measurement positions. The core of the algorithm consists in first calculating the exit wave-field $\psi_{k, j}$ using the Born approximation (equation (1)). The far-field distribution $E_{k, j}$ corresponding to the propagation of $\psi_{k, j}$ is obtained from a Fourier transform operation (denoted as $F$, equation (2)). In equation (3), this quantity is corrected so that the estimated intensity matches the experimental data $I_{j}(\mathbf{q})$. The corrected far-field is then back propagated using inverse Fourier transform operator $\left(F^{-1}\right.$, equation (4)).

The last step (equation (5)) allows the object estimate to be updated, taking into account the probe function and the overlapping condition. The conjugation operation is denoted by ' '. The $\beta$ parameter is the step-size for the current correction $(0<\beta)$ and $\varepsilon$ avoids division by zero. In addition, a support constraint $\chi(\mathbf{r})$ is introduced, leading to the constrained form of the PIE. The expression in equation (5) derives from the minimization of the criterion including the error metric and the penalty term, linked to the constraint ${ }^{8}$. These five operations are performed for the $N$ positions of the illumination function, before the updated object is re-introduced in a new iteration. The whole process is repeated $K$ times. By exchanging the role of the probe and the sample scattering functions in the above formulae, the retrieval of the probe function is obtained.

Data inversion. For the presented result, eight cycles were performed, composed of three iterations over the sample followed by two iterations over the illumination. $\beta$ was set to 0.4 and $\gamma$ to 0.1 . The support constraint allowed the stabilization of the sample position in the computational window. It was introduced as a thin layer box with finite thickness so that the sample height did not extent beyond $50 \mathrm{~nm}$, as determined by atomic force microscopy. The rough shape of the sample estimate is obtained from AFM and SEM observations. A gaussian illumination function was introduced as a first estimate. Its width and height were chosen to provide initially a reasonable agreement with the integrated intensity as measured along $t_{\mathrm{x}}$ or along the rocking curve. In addition, to compensate the measurement-sampling rate, too small along the $\mathbf{q}_{3}$ direction, the retrieved volume was increased by a factor of 2 along the $\mathbf{r}_{3}$ direction. It was made possible owing to the smooth variation of the intensity pattern along the rocking curve, where no interference fringe was expected. The $3 \mathrm{D}$ phase retrieval was enforced to retrieve not only the 
experimentally measured $\left(\mathbf{q}_{1}, \mathbf{q}_{2}\right)$ planes but also the missing planes in between. In equation (3), only the measured intensities were imposed. In this way, the computational window was 60 times larger than the illumination function size along $\mathbf{r}_{1}$ and comparable to it along $\mathbf{r}_{2}$ and $\mathbf{r}_{3}$.

The resolution of the reconstruction was evaluated from the sharpness of the density contrast in the vicinity of the line edge. Along the $x$ and $z$ directions, resolutions of about 4 and 2 pixels were found, which correspond to about $90 \mathrm{~nm}$ and $40 \mathrm{~nm}$, respectively. This approach could not be used along $y$ because of the geometry of the Si lines. In addition, the low scattered intensity of the small line leads to a higher level of noise, which slightly degrades the resolution in this area. Interestingly, we noted that the inversion with support-based algorithms was not possible when only one single set of 3D intensity data was used, except for the measurements performed at the extreme $t_{\mathrm{x}}$ positions. In these cases, only the large line or small line exit field was retrieved.

\section{References}

1. Newton, M. C., Leake, S. J., Harder, R. \& Robinson, I. K. Three-dimensional imaging of strain in a single ZnO nanorod. Nature Mater. 9, 120-124 (2010).

2. Sayre, D. Some implications of a theorem due to Shannon. Acta. Crystallogr. 5, 843-843 (1952).

3. Nugent, K. A. Coherent methods in the X-ray sciences. Adv. Phys. 59, 1-99 (2010).

4. Chamard, V. et al. Strain analysis by inversion of coherent Bragg X-ray diffraction intensity: the illumination problem. J. Mod. Optic. 57, 816-825 (2010).

5. Minkevich, A. A. et al. Inversion of the diffraction pattern from an inhomogeneously strained crystal using an iterative algorithm. Phys. Rev. B 76, 104106-1-5 (2007).

6. Diaz, A. et al. Imaging the displacement field within epitaxial nanostructures by coherent diffraction: a feasibility study. New J. Phys. 12, 035006-1-20 (2010)

7. Vaxelaire, N. et al. 3D strain imaging in sub-micrometer crystals using cross-reciprocal space measurements: Numerical feasibility and experimental methodology. Nucl. Instrum. Meth. B 268, 388-393 (2010).

8. Godard, P., Allain, M. \& Chamard, V. Imaging highly non-homogeneous strain fields in nanocrystals using X-ray Bragg ptychography: a numerical study. Phys. Rev. B 84, 144109-1-11 (2011).

9. Hoppe, W. Beugung im inhomogenen Primaerstrahlwellenfeld. I. Prinzip einer Phasenmessung von Elektronenbeungungsinterferenzen. Acta. Crystallogr. A 25, 495-501 (1969).

10. Rodenburg, J. M. \& Bates, R. The theory of super-resolution electron microscopy via Wignerdistribution deconvolution. Phil. Trans. R. Soc. A 339, 521-553 (1992).

11. Bunk, O. et al. Influence of the overlap parameter on the convergence of the ptychographical iterative engine. Ultramicroscopy 108, 481-487 (2008).

12. Rodenburg, J. M. et al. Hard-X-ray lensless imaging of extended objects. Phys. Rev. Lett 98, 034801-1-4 (2007).

13. Thibault, P. et al. High-resolution scanning X-ray diffraction microscopy. Science 321, 379-382 (2008)

14. Dierolf, M. et al. Ptychographic X-ray computed tomography at the nanoscale. Nature 467, 436-439 (2010).

15. Schropp, A. et al. Non-destructive and quantitative imaging of a nanostructured microchip by ptychographic hard X-ray scanning microscopy. J. Microsc. 241, 9-12 (2010).
16. Giewekemeyer, K. et al. Quantitative biological imaging by ptychographic X-ray diffraction microscopy. Proc. Natl Acad. Sci. USA 107, 529-534 (2010).

17. Faulkner, H. M. L. \& Rodenburg, J. M. Movable aperture lensless transmission microscopy: A novel phase retrieval algorithm. Phys. Rev. Lett. 93, 023903-1-4 (2004).

18. Thibault, P., Dierolf, M., Bunk, O., Menzel, A. \& Pfeiffer, F. Probe retrieval in ptychographic coherent diffractive imaging. Ultramicroscopy 109, 338-343 (2009).

19. Maiden, A. \& Rodenburg, J. M. An improved ptychographical phase retrieval algorithm for diffractive imaging. Ultramicroscopy 109, 1256-1262 (2009).

20. Williams, G. J., Pfeifer, M. A., Vartanyants, I. A. \& Robinson, I. K. Threedimensional imaging of microstructure in Au nanocystals. Phys. Rev. Let. 90, 175501-1-4 (2003).

21. Mastropietro, F. et al. Coherent X-ray wavefront reconstruction of a partially illuminated Fresnel zone plate. Opt. Express 19, 19223-19232 (2011).

22. Paganin, D. M. Coherent X-ray Optics (Oxford University Press, 2006).

23. Schroer, C. G. et al. Coherent X-ray diffraction imaging with nanofocused illumination. Phys. Rev. Lett 101, 090801-1-4 (2008).

24. Takagi, S. A dynamical theory of diffraction for a distorded crystal. J. Phys. Soc. Jpn 26, 1239-1253 (1969).

25. Shi, X., Xiong, G., Huang, X., Harder, R. \& Robinson, I. Structural inhomogeneity in silicon-on-insulator probed with coherent X-ray diffraction. Z. Kristallogr. 225, 610-615 (2010).

26. Chamard, V. et al. Three-dimensional X-ray Fourier transform holography: The Bragg case. Phys. Rev. Lett. 104, 165501-1-4 (2010).

27. Ponchut, C. et al. Photon-counting X-ray imaging at kilohertz frame rates. Nucl. Instrum. Meth. A 576, 109-112 (2007).

\section{Acknowledgements}

We are grateful to P. Ferrand, A. Sentenac and M. Guizar-Sicairos for fruitful discussions and/or careful reading of the manuscript, and to I. Snigireva for the scanning electron microscopy characterizations of the sample. Further atomic force microscopy images were performed by J.-F. Guillet, who is warmly acknowledged. Finally, we thank the ID01 staff (ESRF) for technical support. This work was funded by the French ANR (ANR-08JCJC-0095-01), the FWF Vienna (SFB025), the BMWF Vienna, and the PLATON project of the Austrian Nanoinitiative.

\section{Author contributions}

L.C. provided the sample wafer. J.S. and G.C. fabricated the test pattern and performed the AFM measurements. D.C., A.D, L.C., T.H.M. and V.C. carried out the experiment. F.M. characterized the beam. P.G. and M.A. wrote the algorithm and P.G. analysed the data. V.C. conceived the experiment and wrote the paper with contributions from all.

\section{Additional information}

Competing financial interests: The authors declare no competing financial interests.

Reprints and permission information is available online at http://npg.nature.com/ reprintsandpermissions/

How to cite this article: Godard, P. et al. Three-dimensional high-resolution quantitative microscopy of extended crystals. Nat. Commun. 2:568 doi: 10.1038/ncomms1569 (2011) 